\title{
Brain metastases of neuroendocrine tumor with unknown primary location - Case report
}

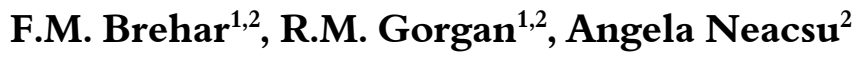 \\ 1"Carol Davila" University of Medicine and Pharmacy Bucharest, Faculty of \\ Medicine \\ 2“Bagdasar-Arseni” Emergency Clinic Hospital, Department of Neurosurgery, \\ Bucharest, Romania
}

\begin{abstract}
Neuroendocrine tumors are tumors derived from the cells of the neuroendocrine system. The majority of metastases of neuroendocrine tumors occur in liver, lungs and bone. The brain is an uncommon site of metastasize for this type of tumor. The authors of this paper present a case of brain metastases of neuroendocrine tumor with undetermined primary location. The patient, a 35 yearsold man, was admitted in our clinic with headache, nausea, vomiting and a mild right facial paresis. Head CT scan and cerebral MRI identified two lesions: one larger lesion with mixed solid and cystic components located in the left basal ganglia and thalamus and a second cystic lesion located deep in the right parietal lobe. All complementary investigations (including thoracic CT scan and whole-body MRI) failed to reveal the primary tumor location. Due to the high vital-risks associated with the open surgical procedure, the patient and his family chose the less invasive procedure of stereotactic biopsy. Postoperatively the patient had no additional neurologic deficits, presenting only the initial mild right central facial paresis. The result of immunohistochemistry examination was cerebral metastases of neuroendocrine
\end{abstract}

tumor. The patient was directed to the Institute of Oncology Fundeni for further investigations and therapeutically management. In conclusion, even if these are rare tumors which rarely metastasize in the brain, the neurosurgeons should take in consideration this pathology when they examine a patient with multiple cerebral lesions with unknown location of primary tumor.

Key words: neuroendocrine tumors, stereotactic biopsy, immunohistochemistry.

\section{Introduction}

Neuroendocrine tumors are tumors derived from the cells of the neuroendocrine system which have the primary role of synthesizing biogenic amines and peptidic hormones (20). The cells of neuroendocrine system could be classified in two distinct cells populations: 1. Nerve cells - located either in central nervous system or peripheral nerve ganglions, which belong to the peptidergic and adrenergic cells of autonomous nervous system and 2. Epithelial cells - in the parenchyma of endocrine glands, but many cells are also disseminated throughout the mucosa of respirator and gastrointestinal tract. Neuroendocrine nerve cells rarely undergo hyperplasia or 
neoplasic transformation, but in epithelial neuroendocrine cells, the neoplasic transformation can occur both in endocrine glands and at the level of teguments and mucosa (17). World Health Organization (WHO) classifies the neuroendocrine tumors according to their proliferation index (PI) into the following groups: well differentiated endocrine tumors ( $\mathrm{PI}<2 \%$ ), well differentiated endocrine carcinomas $(2 \% \leq \mathrm{PI}<15 \%)$, poor differentiated endocrine carcinomas (PI $\geq 15 \%) \quad(12)$. According to the cell type and the peptides synthesized by the cells, there are two main groups of endocrine tumors: pancreatic and carcinoid neuroendocrine tumors (8). The carcinoid neuroendocrine tumors are: tymic carcinoid, bronchial carcinoid, gastric carcinoid, duodenal and pancreatic carcinoid, carcinoid of the jejunum, ileum and proximal colon, distal colon and rectum (18). Neuroendocrine tumors usually metastasize in liver, lungs and bone (10). The brain is an uncommon site of metastasize for this type of tumor. The authors of this paper present a case of rare cerebral metastases of neuroendocrine tumor with undetermined primary location.

\section{Case presentation}

The authors present a case of 35 yearsold man without significant associated pathology who was admitted in our clinic with headache, nausea, vomiting and a mild right facial paresis with acute onset. The neurological exam at presentation demonstrated Romberg's sign positive and a mild right central-type facial paresis. Head CT scan showed two enhanced hyperdense lesions. One larger lesion located in left thalamus and basal ganglia with a mild mass effect on the third and left lateral ventricle. The smaller lesion was located paraventricular, deep-seated in the right parietal lobe. The cerebral MRI showed mixed solid and cystic components of the larger lesion with gadolinium enhancement and mass effect on the ventricular system. The smaller lesion showed only a cystic component without solid nodule. Because of the presence of multiple cerebral lesions, we hypothesized the diagnosis of cerebral metastases and recommended the wholebody MRI which failed to identify the primary lesions. The abdominal echography and thoracic CT scan were also negative. Patient refused to undergo the more invasive investigations like gastro- and colonoscopy. Therefore, all the mentioned investigation failed to reveal the primary source. Due to the high vital-risks associated with the open surgical procedure, the patient and his family chose the less invasive procedure of stereotactic biopsy. The blood tests ruled out any abnormalities in the coagulogram or platelet count. The corticosteroid therapy (dexamethazone 8 $\mathrm{mg}$ intravenous twice daily) had been initiated before the surgical procedure. For this procedure, we used the Leksell stereotactic system and the software Stereotactic Planning System (SPS), NTPS 8.2. The coordinate frame was applied using local anesthetics lidocaine 1\%. The MRI 1,5 Tesla (Philips Integra) (gadolinium-enhanced T1-weighted, $1 \mathrm{~mm}$ slice thickness and T2-weighted, $2 \mathrm{~mm}$ slice thickness) was used for scanning the fiducial system. For operatory procedure, we employed general anesthesia. We chose to target the larger lesion located in left thalamus and insula, because this lesion had both cystic and solid components even if it had a mass effect on the third and left lateral ventricle. 

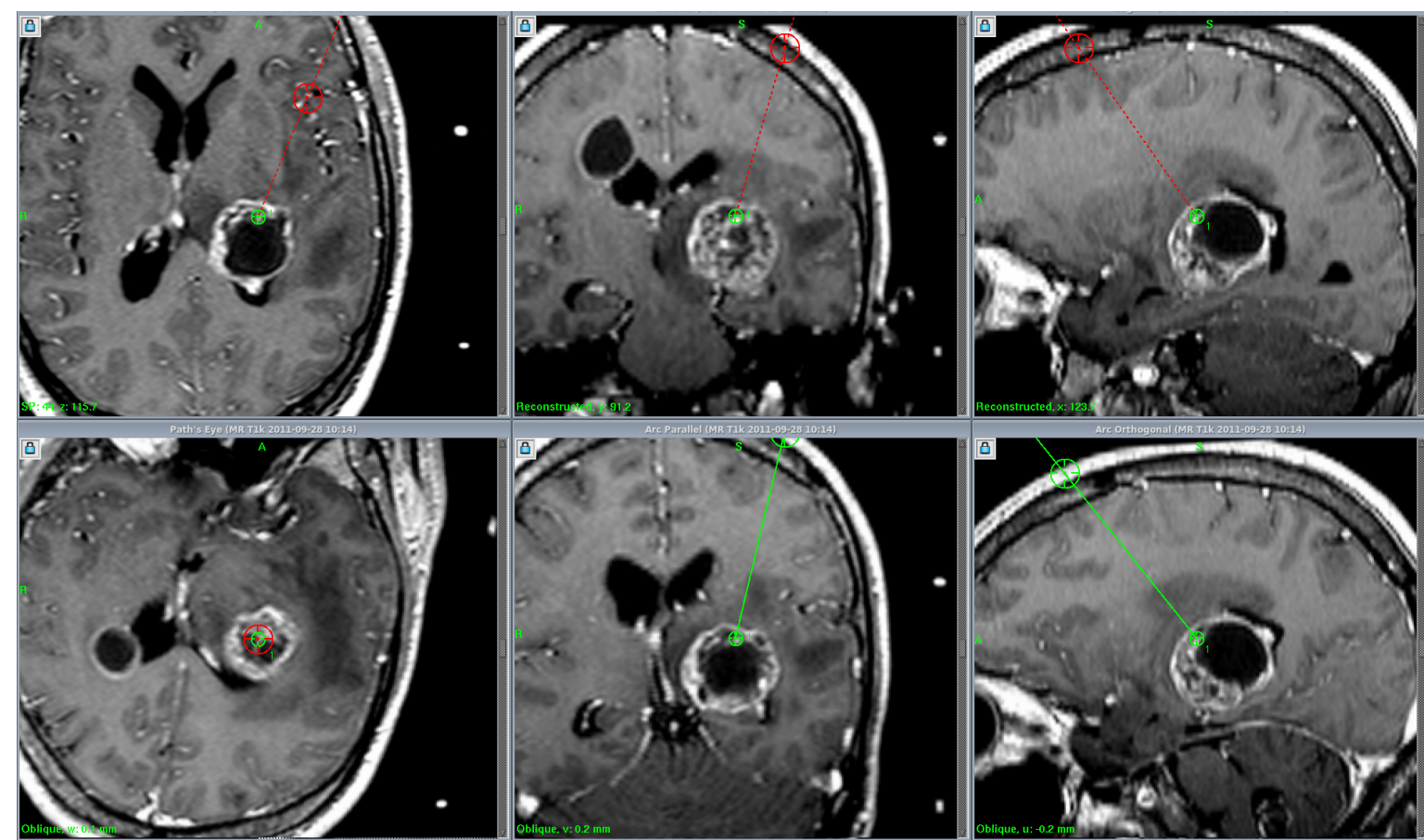

Figure 1 Image of the preoperative planning of the biopsy procedure.

The target is at the gadolinium enhanced periphery of the larger lesion

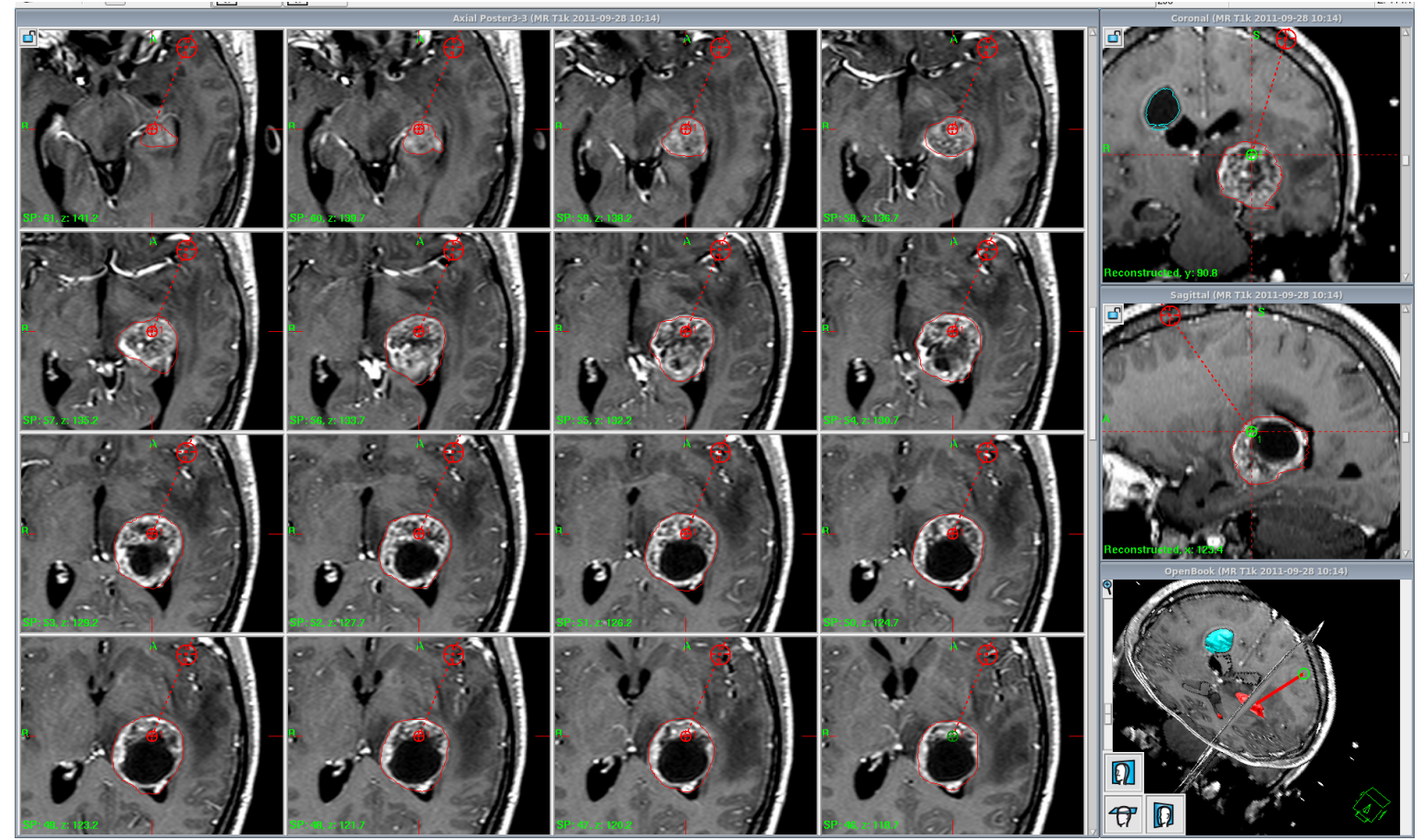

Figure 2 Volumetric assessment of the cerebral lesions. Multiplanar and 3D images of the trajectory 


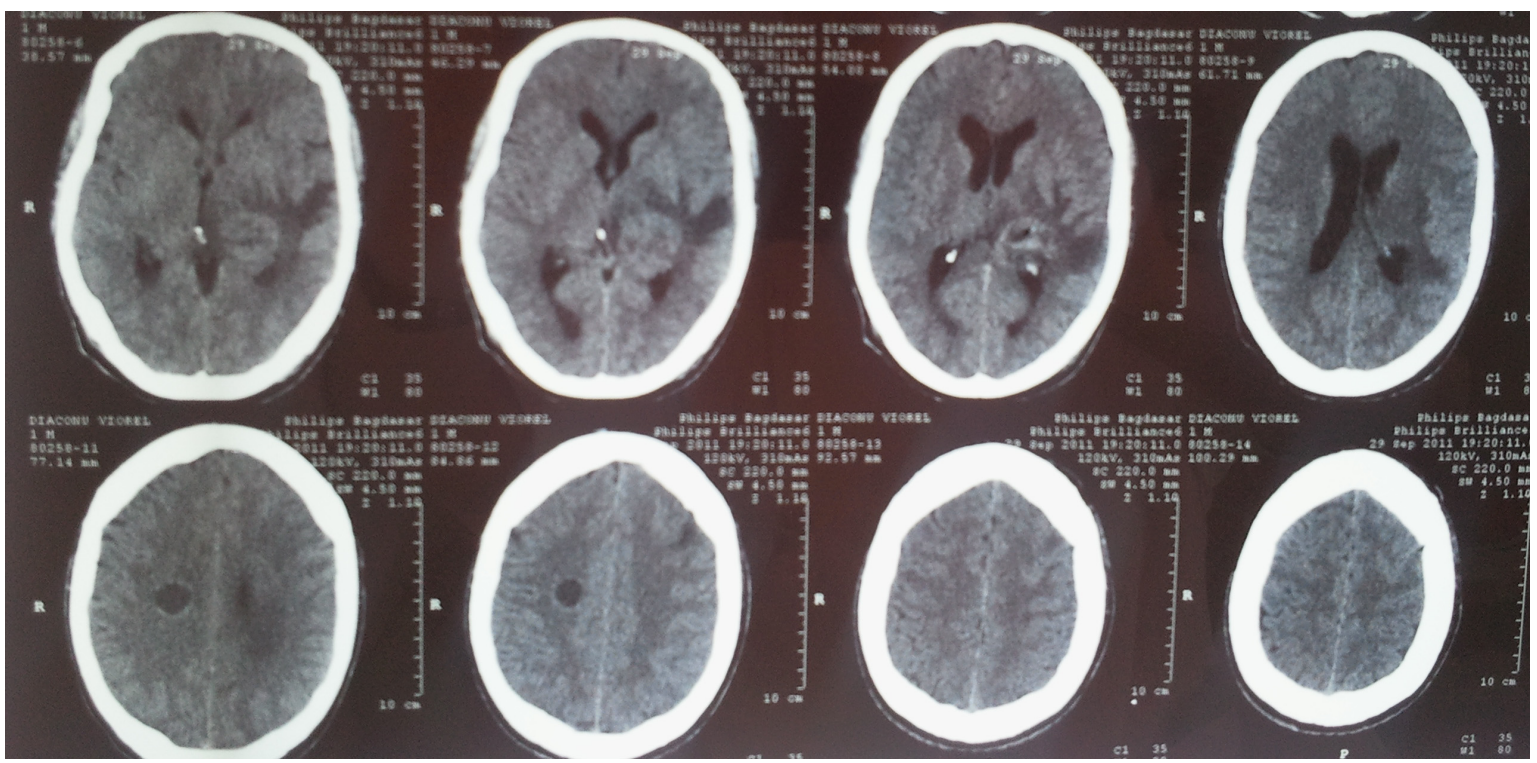

Figure 3 Postoperative cerebral CT scan. No sign of hemorrhage at the biopsy site

A small incision (2 cm long) and a single burr hole located at the left pre-coronal frontal level have been performed. We chose multiple targets located along the shortest trajectory which avoids eloquent areas and vessels. The first target was at the gadolinium enhanced periphery of the lesion (Figure1, Figure 2). Five tumor samples of millimeter size were obtained using the side-cutting Sedan I biopsy needle, which were sent to the department of histopathology. Then we advanced 12 $\mathrm{mm}$ along the trajectory inside the tumor cyst and evacuated approximately $15 \mathrm{ml}$ of a xantocromic appearance fluid. Postoperatively the patient had no additional neurologic deficits, presenting only the initial mild right central facial paresis. The initial symptoms of intracranial hypertension remitted after medical therapy. The patient received corticotherapy, prophylactic antibiotics and prophylactic anticonvulsivant therapy until he was discharged from our department at four days after stereotactic procedure. The postoperative CT scan showed no sign of hemorrhage at the biopsy site and almost complet evacuation of the content of the tumoral cyst (Figure 3).

The histopathological result (hematoxylin \& eosine staining) revealed the diagnosis of metastasis of microcellular carcinoma and recommended the immunohistochemistry test for a more accurate diagnosis. The result of immunohistochemistry examination was cerebral metastases of a neuroendocrine tumor with an undetermined primary location. The patient was directed to the Institute of Oncology Fundeni for further investigations and therapeutically management.

\section{Discussions}

The majority of metastases of neuroendocrine tumors occur in the liver, lungs and bone. Brain location of metastases of neuroendocrine tumor is rare (10). Of all brain metastases, only 1.3-1.4\% have origin in a neuroendocrine tumor (9). In patients with neuroendocrine tumors, 
the incidence of brain metastases is estimated to be $1.5-5 \%$ (6). Of all type, bronchopulmonary neuroendocrine tumors appear to be the most frequent source of cerebral metastases (1). Thus, in the largest series of brain metastases the primary tumor was located in the bronchi or lungs in 45 and $71 \%$ of the patients, respectively (14). In our case, the head CT scan and cerebral MRI identified two lesions. One larger lesion with mixed solid and cystic components located in the left basal ganglia and thalamus and a second cystic lesion located deep in the right parietal lobe. The presence of two cerebral lesions raised the following possible diagnosis: multiple metastases, multiple brain abscesses or multicentric gliomas. In order to confirm the diagnosis of cerebral metastases, we investigated the patient for common tumor primary location like lung, prostate, digestive tract, with thoracic CT scan and whole-body MRI. The patient refused the more invasive investigation procedures like gastro- and colonoscopy. All investigation failed to reveal the primary source. Therefore, obtaining tissue samples from cerebral lesions became mandatory for the therapeutically management of the patient. There were two surgical options in this case. One was the open surgical procedure, which had the advantage of performing a large resection of the tumor, besides obtaining tissue samples. The main limitation of the open procedure was that it would have been impossible to remove both lesions in one surgical session. Moreover, the patient and his caregivers refused the open surgical procedure because of the risk of the postoperative neurological deficits. Therefore, the only procedure which could have obtained a tissue sample with low risks for the patient was the stereotactic biopsy. The availability of image-based stereotactic surgery opened new perspectives for the management of brain tumors. The most common stereotactic procedure became biopsy (4) and one main indication of this procedure was the biopsy of cerebral metastases with unknown primary sources (5). The full protocol of stereotactic biopsy was described by the authors elsewhere (2). Choosing the target is one of the most important parts of the stereotactic procedure because this will aid in obtaining the most representative tissue samples for diagnosis. In this case, there were two lesions accessible for stereotactic biopsy. One larger lesion located in the dominant hemisphere with mass effect on the ventricular system and a second lesion located paraventricular in the right parietal lobe. Choosing the target inside the smaller lesion located on the right side would have been more safely for the patient. But that lesion was cystic and the major risk would have been to not obtain a representative tissue for diagnosis. Therefore, we had to target the larger lesion located in left thalamus and insula, even if that lesion had a mass effect on the third and left lateral ventricle. The result of the classic histopathologic was metastases of microcellular carcinoma. However, in this case, the immunohistochemistry established the final diagnosis of cerebral metastases of neuroendocrine tumors. The abdominal echography, thoracic CT scan and whole-body MRI were not able to reveal the primary source of tumors. On the other hand, the gold standard in the diagnosis of neuroendocrine tumors and their metastases is the scintigraphy of somatostatin receptors (Octreoscan) (13; 15). This is a "whole body" scan, which 
may help not only in diagnosis but also in stadialization of the disease if the tumors expressed somatostatin receptors. Another important investigation is positron emission tomography (PET) (3). The most used tracer is F18 - deoxiglucoza (FDG-PET), but the well differentiated neuroendocrine tumors do not uptake the FDG very well and 68Ga-DOTATATE-PET could be better for detecting pulmonary neuroendocrine tumors (7). PET is able to detect smaller tumors compared with CT or MRI. When the patient is scanned using scintigraphy of somatostatin receptors and PET, the primary tumor is identified in the most cases. Thus, according to one review of over 35,000 patients diagnosed with neuroendocrine tumors over a 31-year period a primary site could not be found or classified in only 13 percent (19). It is important to mention that only a minority of patients present with brain metastases as the initial symptom of their disease or as the only metastatic site $(9 ; 11 ; 16)$. Both the scintigraphy of somatostatin receptors and PET were not available in our clinic. Therefore, we directed the patient to the Institute of Oncology Fundeni for further investigations and oncological treatment.

\section{Conclusions}

The authors present a rare case of cerebral metastases of neuroendocrine tumor with unknown location of the primary lesion. Neuroendocrine tumors could be very small lesions located anywhere along the respirator or digestive tract and even the most advanced investigation tools like whole-body MRI and PET could fail to reveal the primary location of the tumor. Even if these are rare tumors which rarely metastasize in the brain, the neurosurgeon should take in consideration this pathology as a possible diagnostic when he or she is confronted with a case of multiple cerebral lesions with unknown location of primary tumor.

\section{References}

1. Beasley MB, Thunnissen FB, Brambilla E, Hasleton P, Steele R, Hammar SP, Colby TV, Sheppard M, Shimosato Y, Koss MN, Falk R, Travis WD: Pulmonary atypical carcinoid: predictors of survival in 106 cases. Hum Pathol 2000; 31: 1255-1265

2. Brehar, F.M., Gorgan, R.M. Lisievici, M, Imageguided stereotactic biopsy of infiltrative, multicentric and deep-seated supratentorial cerebral gliomas, Romanian Neurosurgery 2012, Volume 19, Issue 2, Pages 87-95

3. Bushnell DL, Baum RP, Standard imaging techniques for neuroendocrine tumors, Endocrinol Metab Clin North Am. 2011 Mar;40(1):153-62

4. Gildenberg PL, Franklin P. Survey of CT-guided stereotactic surgery. Appl Neurophysiol 1985; 48:47780

5. Greenberg Mark S, Handbook of Neurosurgery, Seventh Edition, N.Y., Thieme, 2010

6. Hlatky R, Suki D, Sawaya R: Carcinoid metastasis to the brain. Cancer 2004; 101: 2605-2613

7. Kayani I, Conry BG, Groves AM, Win T, Dickson J, Caplin M, Bomanji JB, A comparison of $68 \mathrm{Ga}-$ DOTATATE and 18F-FDG PET/CT in pulmonary neuroendocrine tumors, J Nucl Med. 2009 Dec;50(12):1927-32

8. Klimstra DS, Modlin IR, Coppola D, Lloyd RV, Suster S. The pathologic classification of neuroendocrine tumors: a review of nomenclature, grading, and staging systems. Pancreas. 2010;39:707-712 9. Maiuri F, Cappabianca P, Del Basso De Caro M, Esposito F: Single brain metastases of carcinoid tumors. J Neurooncol 2004; 66: 327-332

10. Marianne Pavel, Ashley Grossman, Rudolf Arnold et al, ENETS Consensus Guidelines for the Management of Brain, Cardiac and Ovarian Metastases from Neuroendocrine Tumors, Neuroendocrinology 2010;91:326-332

11. Nakamura Y, Shimokawa S, Ishibe R, Ikee T, Taira A: Pulmonary carcinoid found in a patient who presented with initial symptoms of brain metastasis: report of a case. Surg Today 2001; 31: 510-512

12. Oberg K, Castellano D. Current knowledge on diagnosis and staging of neuroendocrine tumors. Cancer Metastasis Rev. 2011;30 Suppl 1:3-7

13. Olsen JO, Pozderac RV, Hinkle G, Hill T, O'Dorisio TM, Schirmer WJ, Ellison EC, O'Dorisio MS., Somatostatin receptor imaging of neuroendocrine 
tumors with indium-111 pentetreotide (Octreoscan), Semin Nucl Med. 1995 Jul;25(3):251-61

14. Patchell RA, Posner JB: Neurologic complications of carcinoid. Neurology 1986; 36:745-741

15. Penz M, Kurtaran A, Vorbeck F, Oberhuber G, Raderer M: Case 2: myocardial metastases from a carcinoid tumor. J Clin Oncol 2000;18: 1596-1597

16. Porter DG, Chakrabarty A, McEvoy A, Bradford R: Intracranial carcinoid without evidence of extracranial disease. Neuropathol Appl Neurobiol 2000; 26: 2983009

17. Ronald A. DeLellis, The Neuroendocrine System and Its Tumors, Am J Clin Pathol 2001;115 (Suppl 1):S5-S16
18. William D Travis, Anthony A Gal, Thomas V Colby, David S Klimstra, Roni Falk, Michael N Koss, MD Reproducibility of neuroendocrine lung tumor classification, Human Pathology, Volume 29, Issue 3 , Pages 272-279, March 1998

19. Yao JC, Hassan M, Phan A, Dagohoy C, Leary C, Mares JE, Abdalla EK, Fleming JB, Vauthey JN, Rashid A, Evans DB, One hundred years after "carcinoid": epidemiology of and prognostic factors for neuroendocrine tumors in 35,825 cases in the United States, J Clin Oncol. 2008;26(18):3063

20. Zulch K.J., Brain tumors. Their biology and pathology. Berlin: Springer-Verlag; 1986. 